\title{
Reizexpositionsbehandlung der Alkoholabhängigkeit Ein Behandlungsmodell zur Rückfallprophylaxe
}

\author{
Sabine Loeber \\ Zentralinstitut für Seelische Gesundheit, Mannheim \\ (Direktor: Prof. Dr. A. Meyer-Lindenberg)
}

In den vergangenen Jahren konnten im Bereich der pharmakologischen Therapie von Suchterkrankungen große Fortschritte erzielt werden (7, 9). Daneben ist auch im Bereich der psychotherapeutischen Behandlung eine enorme Entwicklung zu verzeichnen (6).

Die Rückfallprophylaxe nimmt bei der Behandlung von Abhängigkeitserkrankungen einen zentralen Stellenwert ein. Mittels psycho- und soziotherapeutischer Maßnahmen möchte man den Betroffenen eine bessere Bewältigung ihres Alltags auch ohne Suchtmittel ermöglichen. Zudem weisen aktuelle Modelle des Rückfallprozesses darauf hin, dass es über diese Interventionen hinaus erforderlich ist, auch die langfristig veränderte Reaktion des Patienten auf alkoholassoziierte Reize, wie z. B. den Anblick oder den Geruch des bevorzugten alkoholischen Getränks, therapeutisch zu bearbeiten. Neurobiologische bzw. -psychologische Modelle des Rückfalls gehen von einem antizipatorischen Lerneffekt aus, der nur schwer zu vergessen ist. Deshalb sollte Suchtbehandlung auch darauf abzielen, dass die in entsprechenden kritischen Situationen bestehenden Konditionierungs- bzw. Sensitivierungsprozesse nicht zu einem Rückfall führen.

\section{Modelle des Rückfalls}

\section{Klassische Konditionierung}

Lerntheoretische Rückfallmodelle gehen davon aus, dass Reize, die häufig mit dem Substanzkonsum assoziiert waren, wie z. B. der Geruch des bevorzugten alkoholischen Getränks, im Laufe der Zeit bei einer Person klassisch konditionierte Reaktionen, eine sogenannte Cuereactivity, auslösen können. Diese Reaktionen spiegeln sich sowohl in emotionalen-motivationalen als auch in psychophysiologischen Veränderungen wider und werden vom Betroffenen eventuell bewusst als Alkoholverlangen erlebt. Es existieren verschiedene Modelle zur Natur
Die Verbesserung der suchtmedizinischen Versorgungssituation und die weitere Entwicklung effektiver Behandlungsverfahren erscheinen angesichts aktueller Schätzungen der Anzahl von Menschen mit Alkoholproblemen als eine wichtige Aufgabe. So wird in neueren Untersuchungen die Zahl der alkoholabhängigen Erwachsenen in Deutschland (12-Monats-Prävalenz) auf mehr als 1,7 Millionen (2,4\%) geschätzt (2). Ein „schädlicher Gebrauch“ liegt nach diesen Berechnungen bei etwa weiteren 1,7 Millionen Erwachsenen vor. Ein neuer therapeutischer Ansatz stellt die Reizexpositionsbehandlung für alkoholabhängige Patienten dar, welche die Ergebnisse aus der neurobiologischen Grundlagenforschung aufgreift. Die Ergebnisse einer Therapievergleichsstudie verdeutlichen das Potenzial, aber auch die Grenzen dieses Ansatzes.

dieser Reaktionen. Gemeinsam ist diesen, dass alkoholspezifische Stimuli auch nach längerer Abstinenz vermittelt über Prozesse der Konditionierung Reaktionen hervorrufen, welche die Auftretenswahrscheinlichkeit eines Rückfalls erhöhen.

Neuronale Adaptation

Neuere biochemische Erklärungsansätze des Alkoholverlangens postulieren bei einem ständigen Alkoholkonsum eine neuronale Adaptation (Sensitivierung) des dopaminergen Belohnungssystems (8). Diese soll sich in einer erhöhten elektrisch bedingten Ausschüttung von Dopamin und Acetylcholin im mesolimbischenmesokorticalen Bereich bei Präsentation alkoholbezogener Stimuli ausdrücken. Dadurch kann es zu verstärktem Alkoholverlangen und damit zu einer erhöhten Rückfallgefahr kommen. Dabei ist eine deutliche Modulation durch situative Bedingungen zu beobachten. Die erhöhte Alkoholsensitivität ist in früheren Trinksituationen am stärksten ausgeprägt.

\section{Training durch Reizexposition}

Ausgehend von diesen Überlegungen erscheint die Konfrontation des alkoholabhängigen Patienten mit kritischen alkoholassoziierten Reizen ohne dass der Patient tatsächlich Alkohol 
konsumiert, eine vielversprechende Möglichkeit zur Reduktion von Alkoholverlangen und Rückfallhäufigkeit. Durch dieses Verfahren, dass in ähnlicher Weise in der Angst- und Zwangsbehandlung zur Löschung von Reaktionen auf aversive Reize sehr erfolgreich eingesetzt wird, könnte der Patient den Umgang mit konditionierten Reaktionen wie beispielsweise dem Alkoholverlangen üben. Über mehrere Sitzungen sollte es zu einer Löschung der konditionierten Reaktionen kommen. Die Ergebnisse von Untersuchungen aus dem angloamerikanischen Sprachraum unterstützen die Effektivität einer solchen Reizexpositionsbehandlung (1). Bislang erfolgten jedoch keine Therapievergleichsuntersuchungen, bei denen die Reizexpositionsbehandlung einer bewährten psychotherapeutischen Behandlung von Abhängigkeitserkrankungen gegenübergestellt wurde.

\section{Evaluation der Reizexposition}

In der Klinik für Abhängiges Verhalten und Suchtmedizin am Zentralinstitut für Seelische Gesundheit in Mannheim wurde 2001 die Reizexpositionsbehandlung als Alternative zu einem kognitiv-verhaltenstherapeutischen Behandlungsprogramm etabliert. Im Rahmen einer Therapievergleichsstudie sollte überprüft werden, ob es sich bei der Reizexpositionsbehandlung um einen neuen Behandlungsansatz für alkoholabhängige Patienten handelt, der im Vergleich zur bisherigen Behandlung - einem kognitiv-verhaltenstherapeutischen Ansatz zu vergleichbaren oder besseren Ergebnissen führt. Die Behandlung alkoholabhängiger Patienten erfolgte im Rahmen einer dreiwöchigen, qualifizierten Entzugsbehandlung, die neben der medizinischen und sozialen Betreuung der Patienten eine psychotherapeutische Behandlung beinhaltete.

Sowohl bei der kognitiv-verhaltenstherapeutischen als auch der Behandlung durch Reizexposition stand die Auseinandersetzung des
Patienten mit individuellen, kritischen Rückfallsituationen und die Erarbeitung alternativer Verhaltensstrategien zum Umgang mit diesen Situationen im Mittelpunkt. Im Rahmen des Reizexpositionstrainings nahmen die Patienten in einem Zeitraum von drei Wochen an neun ca. zweistündigen Reizexpositionssitzungen teil. Hier lernten diese, sich besonders kritischen Situationen auszusetzen, d. h. sich mit ihrem gewohnten alkoholischen Getränk zu konfrontieren (sehen, riechen). Dies geschah z. B. in vivo durch das konkrete Aufsuchen solcher Situationen (Kneipenbesuch, Einkauf im Supermarkt etc.). Das Ziel dieser Sitzungen besteht darin, dass die Patienten erleben, wie sich ihr Verlangen nach Alkohol entwickelt, welche körperlichen Reaktionen und Gedanken auftreten und wie sich das Verlangen über die Zeit entwickelt. Die Patienten erfahren dabei, welche Möglichkeiten ihnen zur abstinenten Bewältigung zur Verfügung stehen. Die Sitzungen finden als Einzelsitzungen oder in Kleingruppen statt. Die kognitiv-verhaltenstherapeutische Behandlung erstreckte sich ebenfalls über einen Zeitraum von drei Wochen und wurde von denselben Therapeuten durchgeführt. Es fand jedoch keine Konfrontation mit dem bevorzugten alkoholischen Getränk statt. Alternative Verhaltensstrategien wurden im Rollenspiel geübt. Für weitere Details zur Reizexpositionsbehandlung als auch der kognitiv-verhaltenstherapeutischen Behandlung sei der interessierte Leser auf das Manual von Mann et al. verwiesen (5).

Für eine Evaluation der Reizexpositionsbehandlung erfolgte eine sequenzielle Zuweisung von je 30 alkoholabhängigen Patienten zu der kognitiv-verhaltenstherapeutischen Behandlung oder der Reizexpositionsbehandlung. Sowohl vor als auch nach der Teilnahme am jeweiligen Therapieprogramm wurden die Patienten zur Erfassung der Reizreaktivität psychophysiologisch untersucht. Das Trinkverhalten beider Behandlungsgruppen wurde inner-

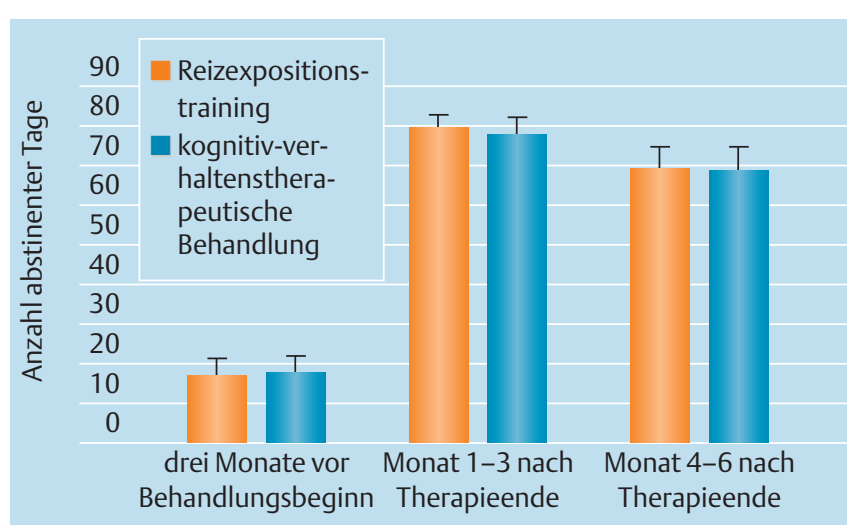

Abb. 1 Anzahl abstinenter Tage vor Therapiebeginn sowie in den Zeiträumen von 1 bis 3 Monaten und 4 bis 6 Monaten nach Beendigung der jeweiligen Behandlung

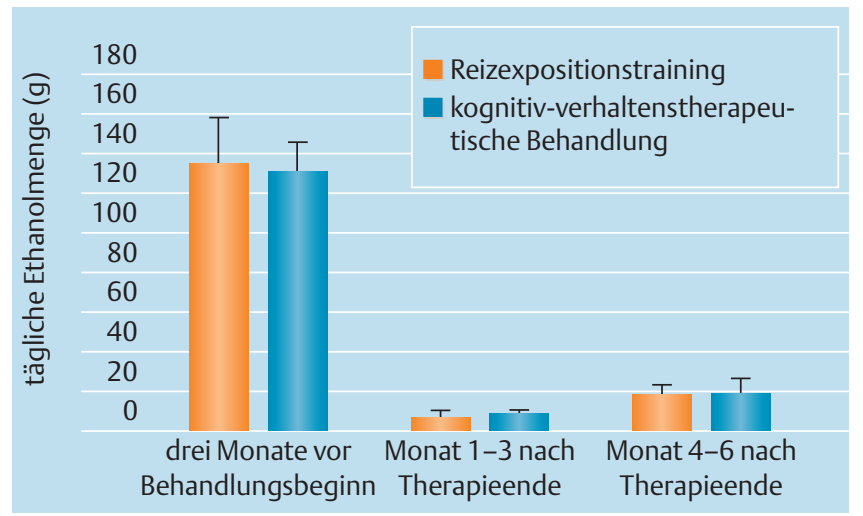

Abb. 2 Tägliche Trinkmengen vor Therapiebeginn sowie in den Zeiträumen von 1 bis 3 Monaten und 4 bis 6 Monaten nach Beendigung der jeweiligen Behandlung 
halb der ersten sechs Monate nach Entlassung aus der stationären Behandlung analysiert.

\section{Therapieeffektivität}

Die Ergebnisse unserer Untersuchung belegen die Wirksamkeit der Reizexpositionsbehandlung. So zeigen sie eine signifikante Reduktion der Anzahl schwerer Trinktage und der täglichen Trinkmenge im Vergleich zu den letzten drei Monaten vor der Behandlung. Eine signifikante Zunahme der Anzahl abstinenter Tage war ebenso zu verzeichnen (3).

Sowohl im Hinblick auf die Rückfallhäufigkeit als auch auf die Variablen des Trinkverhaltens (Anzahl schwerer Trinktage, tägliche Trinkmenge, Anzahl abstinenter Tage) zeigte sich eine vergleichbare Therapieeffektivität der Reizexpositionsbehandlung und der kognitivverhaltenstherapeutischen Behandlung, einem bei Abhängigkeitserkrankungen bewährten Therapieansatz (Abb. 1 und 2).

Die Reizexpositionsbehandlung zeigte im Vergleich zur kognitiv-verhaltenstherapeutischen Behandlung keine Überlegenheit. Dies spiegelte sich u.a. in der Änderung der Reizreaktivität wider, die sowohl vor als auch nach Teilnahme am jeweiligen Behandlungsprogramm erhoben wurde. So konnten wir beispielsweise eine signifikante Reduktion des subjektiv erlebten Alkoholverlangens bei Präsentation alkoholassoziierter Reize im Verlauf der Therapie nachweisen. Dieser Effekt zeigte sich jedoch in beiden Behandlungsgruppen gleichermaßen. Auch führte die Reizexpositionsbehandlung nicht zu einer Abnahme der emotional positiven Verarbeitung alkoholassoziierter Reize (4).

\section{Ausblick}

Die Ergebnisse dieser Untersuchung verdeutlichen, dass die Umsetzung von Grundlagenerkenntnissen zu neurobiologischen Veränderungen bei Abhängigkeitserkrankungen und deren Bedeutung im Rückfallgeschehen in psychotherapeutische Behandlungsverfahren ein komplexes Unterfangen ist. Auch wenn es zunächst gelungen ist, mit der Reizexpositionsbehandlung ein Therapieverfahren zu entwickeln, welches bewährten Therapieansätzen wie der kognitiv-verhaltenstherapeutischen Behandlung zumindest gleichwertig sein könnte, bleibt die Veränderung reizabhängiger Reaktionen und überdauernder Sensitivierungsprozesse nach wie vor eine große Herausforderung. Nichtsdestotrotz könnte die Reizexpositionsbehandlung die Grundlage für zukünftige Therapieansätze werden, bei denen Modifikationen und differenzielle Therapieindikationsstellungen von Bedeutung sein könnten. Hierzu bleiben die Ergebnisse weiterer Forschungsbemühungen abzuwarten.
Cue exposure treatment for alcohol dependent patients - A treatment approach for relapse prevention

In light of current estimations on the number of individuals with a problematic consumption of alcohol, new treatment approaches are warranted. At present, the number of individuals suffering from alcohol dependence in Germany is estimated at more than 1.7 million (2.4\%). An additional 1.7 million (2.4\%) are abusing alcohol. Cue exposure treatment is a new treatment approach derived from findings of basic research on learning mechanisms contributing to substance abuse and dependence. The results of a controlled treatment trial presented here underline the general efficacy as well as the limitations of cue exposure treatment.

\section{Key words}

Addiction - prevention of relapse - exposure to stimulus incentives - conditioning - sensitization - cognitive behaviour therapeutical treatment

\section{Literatur}

1 Conklin CA, Tiffany ST. Applying extinction research and theory to cue-exposure addiction treatments. Addiction 2002; 97 (2): 155-167

2 Deutsche Hauptstelle für Suchtfragen (DHS) e.V. (Hrsg.). Jahrbuch Sucht 2006. Geesthacht: Neuland Verlag, 2006

3 Loeber S, Croissant B, Heinz A et al. Cue exposure in the treatment of alcohol dependence: effects on drinking outcome, craving, and self-efficacy. Br J Clin Psychol 2006; 45 (Pt 4): 515-529

4 Loeber S, Croissant B, Nakovics $\mathrm{H}$ et al. The startle reflex in alcohol-dependent patients: changes after cognitive-behavioral therapy and predictive validity for drinking behaviour. A pilot study. Psychother Psychosom 2007; 76 (6): 385-390

5 Mann K, Loeber S, Croissant B, Kiefer F. Qualifizierte Entzugsbehandlung von Alkoholabhängigen: Ein Manual zur Pharmako- und Psychotherapie. Köln: Deutscher Ärzteverlag, 2006

6 Mann KF, Czisch P, Mundle G. Psychotherapie der Alkoholabhängigkeit. In: Soyka M, Möller HJ (Hrsg.). Alkoholismus bei psychischen Störungen. Heidelberg: Springer, 1997: 119-135

7 Mann K. Pharmacotherapy of alcohol dependence: a review of the clinical data. CNS Drugs 2004; 18 (8): 485-504

8 Robinson TE, Berridge KC. The psychology and neurobiology of addiction: an incentive-sensitization view. Addiction 2000; 95 (Suppl. 2): S91-S117

9 Sass H, Soyka M, Mann KF, Zieglgansberger W. Relapse prevention by acamprosate: Results from a placebo-controlled study on alcohol-dependence. Arch Gen Psychiatry 1996; 53 (8): 673-680

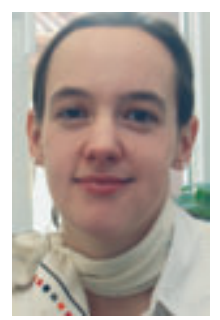

\section{Korrespondenz}

Dr. phil. Sabine Loeber

Zentralinstitut für Seelische

Gesundheit, 15

68159 Mannheim

sabine.loeber@zi-mannheim.de

\section{Danksagung}

Die Autorin möchte an dieser Stelle den Personen danken, die die Entstehung dieser Studie ermöglicht haben, insbesondere Herrn Prof. Karl Mann und Frau Prof. Herta Flor, sowie den Mitarbeiterinnen und Mitarbeitern der Klinik für Abhängiges Verhalten und Suchtmedizin für ihr persönliches Engagement. 